\title{
LABVIEW BASED RF CHARACTERIZATION AND TESTING OF DUAL MODE PHASE SHIFTER
}

\author{
Kirti Bansal $^{1}$, Raj Kumar Gautam ${ }^{2}$, B S Matheru ${ }^{3}$ \\ ${ }^{I}$ Scientist'C', Solid State Physics Laboratory, DRDO, Delhi, India \\ ${ }^{2}$ Scientist'D', Solid State Physics Laboratory, DRDO, Delhi, India \\ ${ }^{3}$ Scientist' $G$ ', Solid State Physics Laboratory, DRDO, Delhi, India
}

\begin{abstract}
R.F. characterization of C-band dual mode ferrite phase shifter cannot be done manually due to enormous and huge amount of data. So this paper provides the solution in term of automated procedure for testing of C-band DMPS in LabVIEW(Laboratory Virtual Instrument Engineering Workbench) software. Dual mode phase shifter is currently being used in phased array Radar and WLR (Weapon Locating Radar). The DMPS is in production to realize the exorbitant demand of these radars. The desirable characteristic of phase shifter for phased array radar as differential phase shift, insertion loss, return loss, Hi-low phase difference, insertion phase, Rx-Tx phase can be measured with high accuracy. The output can be presented in any desirable form such as concluded summary form, graphical form and in excel sheet for future reference purposes.
\end{abstract}

Key words - Dual mode phase shifter, LabVIEW, GPIB, VNA, Hi-Low phase difference, Command generator

\section{INTRODUCTION}

The applications of microwave ferrite phase shifters are numerous, usually the most important application is within a phased array antenna system (electronically steerable array, or ESA), in which the phase of a large number of radiating elements are controlled to force the radiated electromagnetic wave to add up at a particular angle to the array. So in order to achieve production friendly calibrated phase shifter so as to fulfill the enormous demand of weapon locating radar the testing and optimization process of phase shifters should be fully automated and fast [2].

The dual-mode phase shifter is known as a reciprocal ferrite phase shifter. The DMPSs are ideal for passive phased array antenna applications [5]. Basically it is a variant of the Faraday rotation phase shifter. A ferrite phase shifters are being used to provide latching reciprocal phase shift over a moderate frequency band. The phase shift section is inherently a non-reciprocal one. Reciprocal phase shift is achieved by incorporating non-reciprocal polariser at the two ends of the phase shift section (as shown in fig.1). A linearly polarised RF signal incident at the left port is converted to the one sense of circular polarization by the non reciprocal polariser then it passes through the longitudinally magnetized ferrite rod and experiences the phase shift, and is again converted to linear polarization by the second polariser. At the output any undesired orthogonal polarised signal is absorbed by a resistive film[1].

LabVIEW (short name for Laboratory Virtual Instrumentation Engineering Workbench) is a system design platform and development environment for a visual

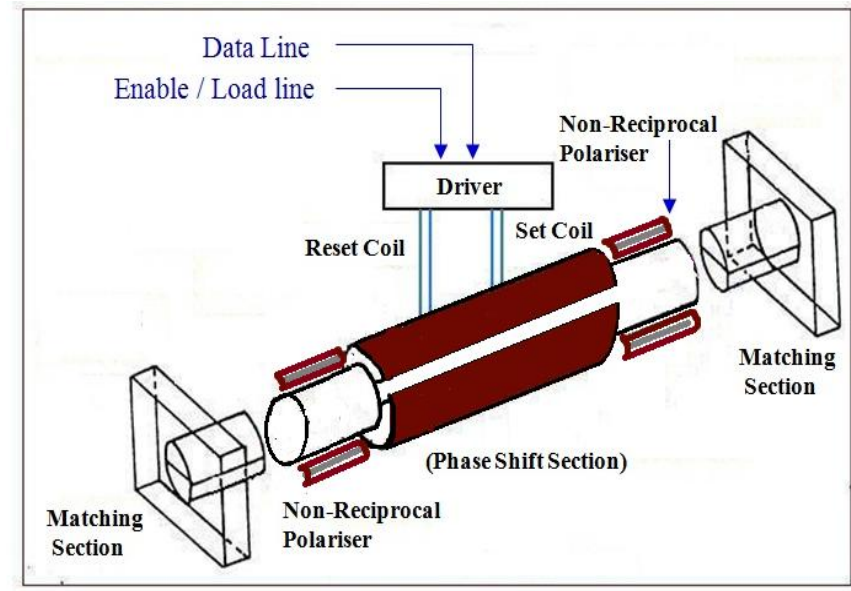

Fig -1: C-band dual mode phase shifter

programming language from National Instruments. The graphical language is named " $G$ ". The code files have the extension ".vi", which is an abbreviation for "Virtual Instrument" [6]. LabVIEW is a development environment for building various applications that interact with realworld data or signals in fields such as science and technology. The net result of using a tool such as LabVIEW is that higher quality projects can be completed in less time with better accuracy. By labVIEW one can:

- Operate the instrumentation program

- Control selected hardware

- $\quad$ Analyze acquired data

- Display results

Large amount of data is required for characterization of phase shifter i.e.

No. of frequencies $=17$

No. of phase states $=128(7 \mathrm{bit})$ 


$$
\begin{aligned}
\text { Data: } & |\mathrm{S} 21|, \angle \mathrm{S} 21, \\
& |\mathrm{~S} 12|, \angle \mathrm{S} 12, \\
& |\mathrm{~S} 11|, \angle \mathrm{S} 11, \\
& |\mathrm{~S} 22|, \angle \mathrm{S} 22
\end{aligned}
$$

Total No. of data points $=8 \times 128 \times 17 \sim 17400$

Therefore, this is a very huge data so cannot be characterized it manually. So ATP is requires to characterized the phase shifter.

The Key highlighted features of the LabVIEW based automated test procedure for complete characterizations of the phase shifter are to make it user friendly and for efficient testing of enormous phase shifters:

- All four S parameter (complete characterization) or single parameter (for fast testing purpose) can be measured in short span of time.

- Comprehensive conclusive summary of all user-defined parameters can be presented. It is essential to avoid the handling of millions of data values.

- For testing purpose, a single command can be given any number of times manually or continuously or in loop automatically.

- Data rate can be changed (i.e. program can be fast or slow according to device requirements).

- For industrial and other technical purposes the output parameters can be presented in graphical format also.

- The front panel is very user friendly. Data can be provided manually or automatically as well as the complete program can be controlled manually or automatically as per user desire.

- Output data can be transferred in to any user specific format. (As excel sheet, notepad etc.)

\section{AUTOMATED TEST PROCEDURE DESCRIPTION AND DESIGN}

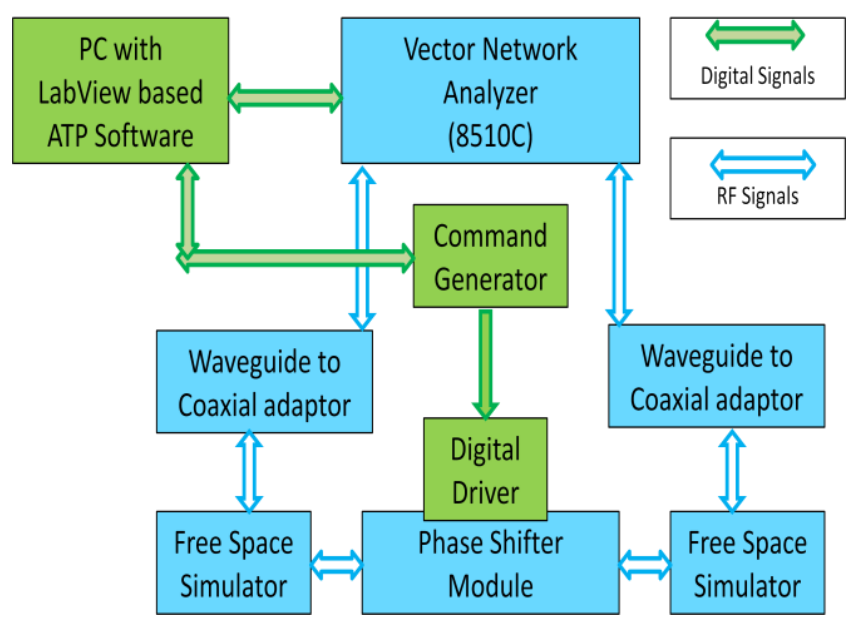

Fig -2: Hardware setup Block diagram
The basic building blocks of complete hardware setup [4]

- Dual Mode Phase Shifter

- Vector Network Analyser

- Computer with GPIB card and LabVIEW software

- Command Generator

Dual Mode Phase Shifter: Dual mode phase shifter (component whose RF Characterization has to be done) is attached with integrated 7-bit digital driver circuit. The digital driver receives the controlling command from Command Generator. Phase shifter receives its RF power from vector network analyser.

Vector Network Analyser: A vector network analyser is a test system that enables the performance evaluation of radio frequency $(\mathrm{RF})$ and microwave devices, to be characterised in terms of S-Parameters. Vector network analyser is initiated by the LabVIEW based ATP software. Then it measures all parameters of phase shifter and transfers to Labview computer to store the data.

Computer with GPIB card and LabVIEW software: A computer is installed with LabVIEW software and GPIB card. The computer is interfaced with the VNA through GPIB port while the computer's parallel port is used as I/O for the Command Generator.

Command Generator: Input command data to the driver circuit of phase shifter is provided by the driver controller or the command generator circuit. The output of the driver goes to the reset / set coils of the phase shifter.

\section{WORKING DESCRIPTION}

An electronic Digital Driver Circuit does the interfacing between the system and the phase shifter. A command generator circuit generates the desired waveforms for driver data input. The digital driver translates the digital commands into appropriate current and voltage Waveforms for Reset \& Set coils of the phase shifter to achieve the desired phase state.

The main set up consists of various instruments/devices; these instruments/devices are connected to LabVIEW software as the main controller for these systems. The first device is the waveform protocol generator i.e. command generator which receives the data from LabVIEW. The Driver Controller processes information and transmits controlling desired signals in to driver of phase shifter unit. It has a user friendly input panel where the user can enter the details like frequency range, no. of points parameters etc which are specified for initialization and calibration, $\mathrm{Cal}$ set, temperature, no. of observation points and then the DUT number. The other one is the vector network analyser whose parameters are automatically set by the LabVIEW software and output data being sent to LabVIEW software for further calculations. The program flowchart is shown in fig 3 . 


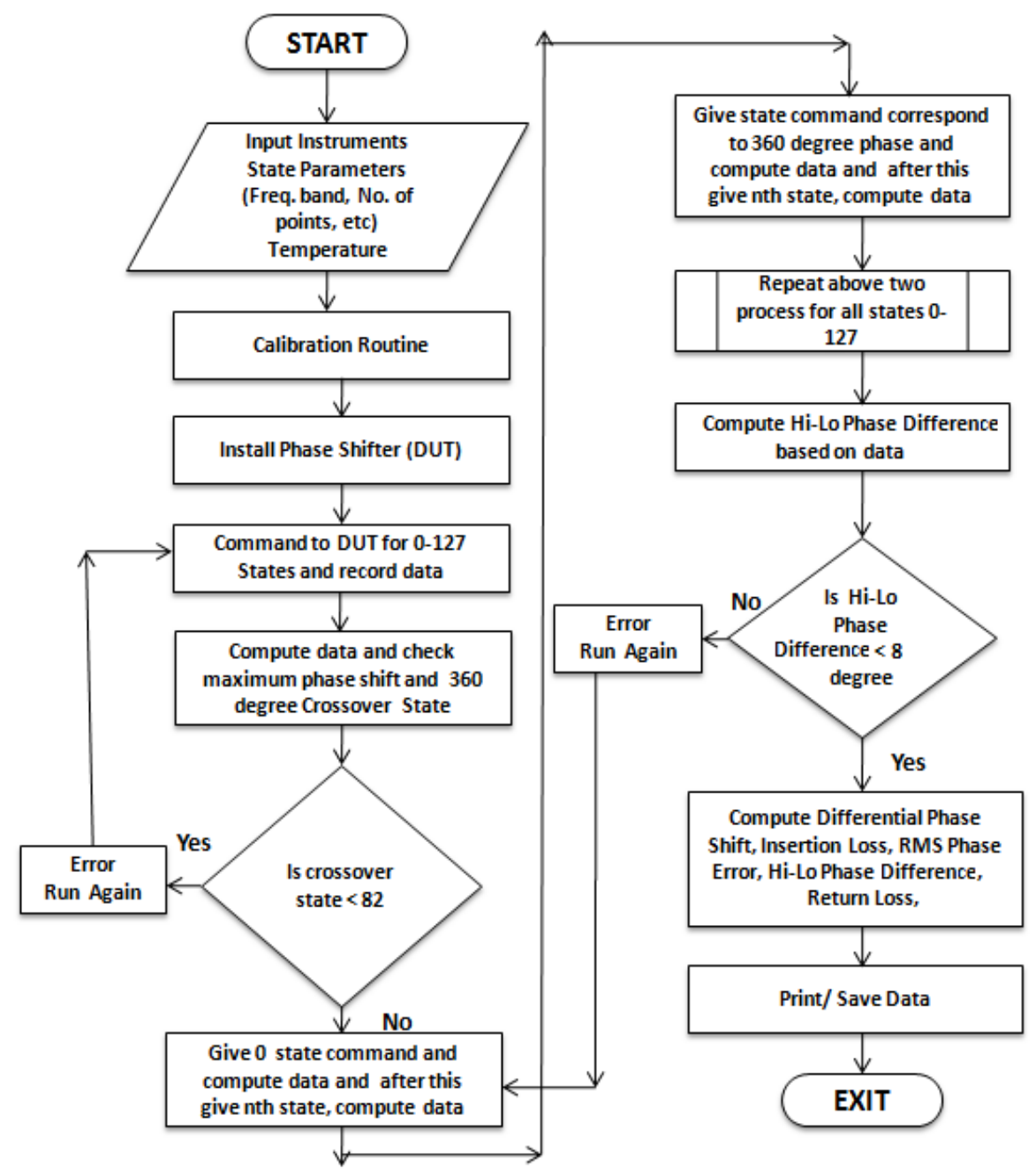

Fig -3: Flow chart of ATP software

The phase shifter is tested by inputting the different phase states from 0 to 127 (for 7 bit control) which correspond to differential phase shift more than 360 degrees and recording the data from the VNA. Then it notes down the state at which 360 degree is crossed and it is known as crossover point. After that it again rotate the phase shifter from $1^{\text {st }}$ state to $\mathrm{n}$ state and last state to $\mathrm{n}$ state and same process repeat for $n+1$ states till 360 degree crossing state and calculate the difference between them to calculate the $\mathrm{Hi}$ Low phase difference. The recorded data set consists of all four S parameters (Mag and Phase) at 128 phase setting and at the specified number of frequency points (17 points, max 801 points) within the given frequency band.

Basically, Hi-Low phase difference shows repeatability of phase shift values in a phase shifter at certain state. It calculates the difference in phase angle between the previous zero degree phase state to specific phase state and previous 360 degree phase state to specific phase state and this loop repeat till 360 degree crossing state.

\section{ATP PROGRAM VI HIERARCHI}

Labview is a graphical language so its programs known as the VI(virtual instrument) and the subprograms are known as the SubVI [3]. The complex block diagram of a Labview VI can be simplified by converting sections of the block diagram into sub VIs. The sub VI can be created just like a VI and then it can be used multiple times in program. One can also:

- Customize the icon for the subVI

- Configure the terminals

- Use it within other VIs repeatedly

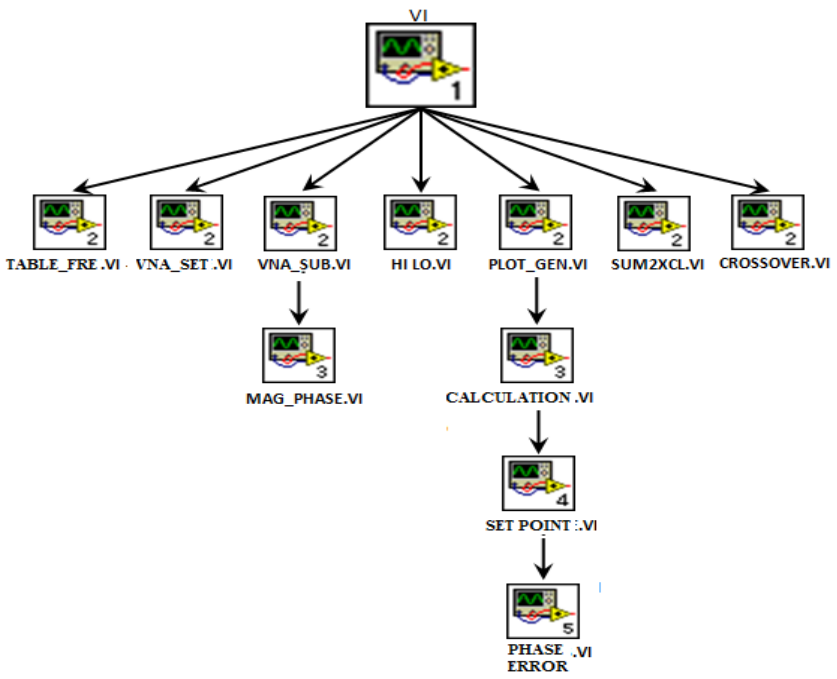

Fig -4: Tree representation of all Main VI and Sub VIs 
As can be seen in fig 4 the main VI consists of multiple subVI and these subVI have their own subVI's. so it completely make a tree.

The functions of the sub VI are as follows:

- TABLE_FRE.VI: The TABLE_FRE.VI generates the various frequency point in the given frequency band at which the phase shifter performance has to be recorded and analyzed.

- VNA_SET.VI: This VNA_SET.VI basically set the different parameters as frequency band, no. of points, format (log, polar), calibrations etc.

- VNA_SUB.VI: This VI reads data at each phase state from the VNA through the GPIB port.

- HI_LO.VI: This VI sends the phase state to calculate HI-LOW phase difference. It calculates the difference in angle between the previous 0 degree phase state to specific phase state and previous 360 degree phase state to specific phase state and this loop repeat till 360 degree crossing state.
- CROSSOVER.VI: This VI records the phase state at which the phase shifter has crossed 360 degree phase angle.

- MAG_PHASE.VI: The data recorded from the VNA is in string format. This VI is used to calculate the magnitude and phase from the string.

- CALCULATION.VI: This VI calculates the desired parameters such as insertion loss, phase shift, RMS phase error, HI-low phase difference, insertion phase etc. from the recorded data.

- $\quad$ SET POINT.VI: A Set point table is generated for computation of RMS phase error.

- $\quad$ PLOT.VI and PRINT.VI: These VIs presents the calculated data in the form of graphs and provides a hard copy of the data respectively.

- SUM_2XCL.VI: The final results are saved in EXCEL sheet here.

The block diagram of Calculation.VI is shown in fig.5. The various parameters can be calculated using this VI. A Mathematical block for implementing of all if and else condition is used here.

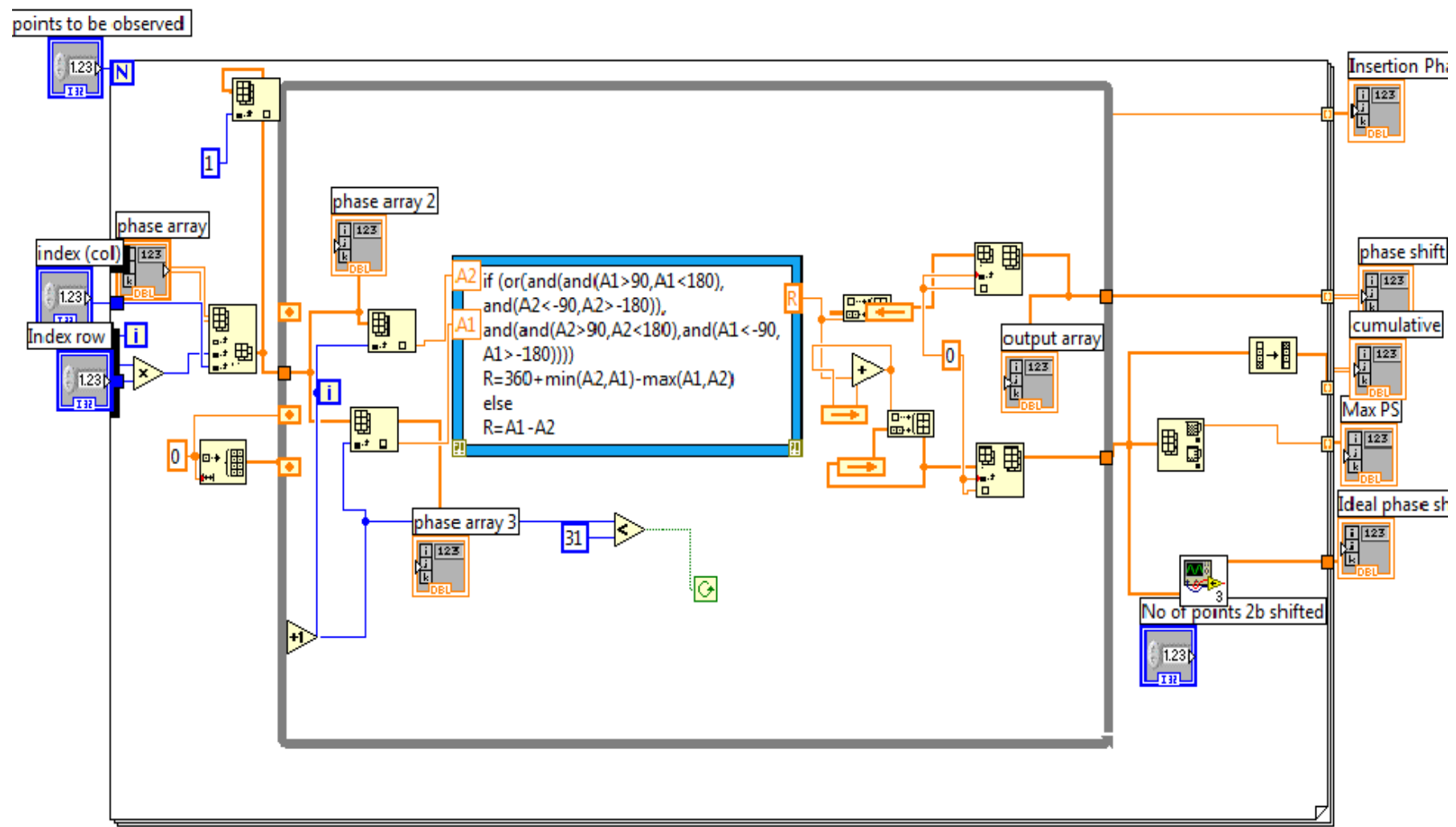

Figure 5: Sub VI for calculating various parameters in DMPS

\section{EXPERIMENTAL RESULTS}

The various user-defined parameters used for complete RF characterization and testing of the phase shifters are given below:

- Phase Shift with Command states

- Average Insertion Loss: This is computed as the arithmetic mean of the transmission coefficients at all phase states at any given frequency, expressed in $\mathrm{dB}$.
- Fluctuation in Average Insertion Loss: This is computed as the square root of the mean of the squares of the difference between the average transmission coefficient and each individual transmission coefficients.

- Average Return Loss: This is computed as the arithmetic mean of the reflection coefficients at all phase states at any given frequency, expressed in $\mathrm{dB}$. 
- Hi-Low phase difference: This is calculated as the difference in angle between the previous 0 degree phase state to specific phase state and previous 360 degree phase state to specific phase state and this loop repeat till 360 degree crossing state.

- Rx-Tx Phase error: This is the measurement of reciprocity of a phase shifter.
- The final automatic test procedure for characterization of the DMPS is designed and developed with aforementioned method. and the following panel shows the result in the front panel as shown in fig. $6 a$ \& fig. 6 b.
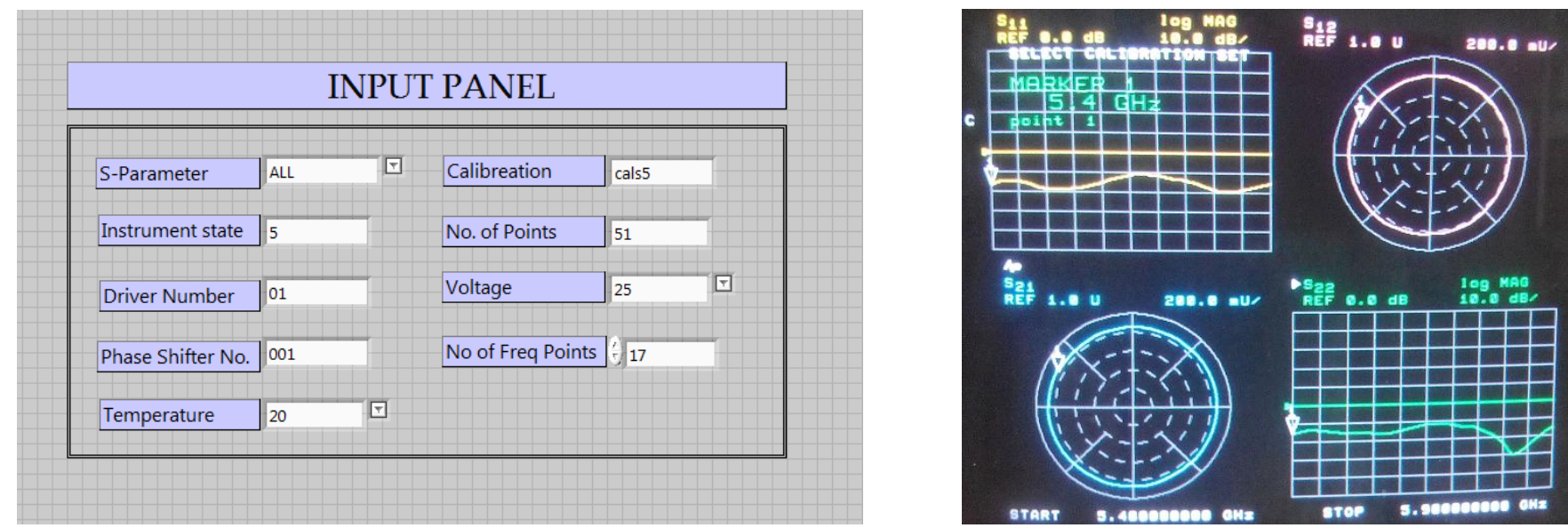

Fig 6a: User input interface panel and S-Parameter interpretation on VNA

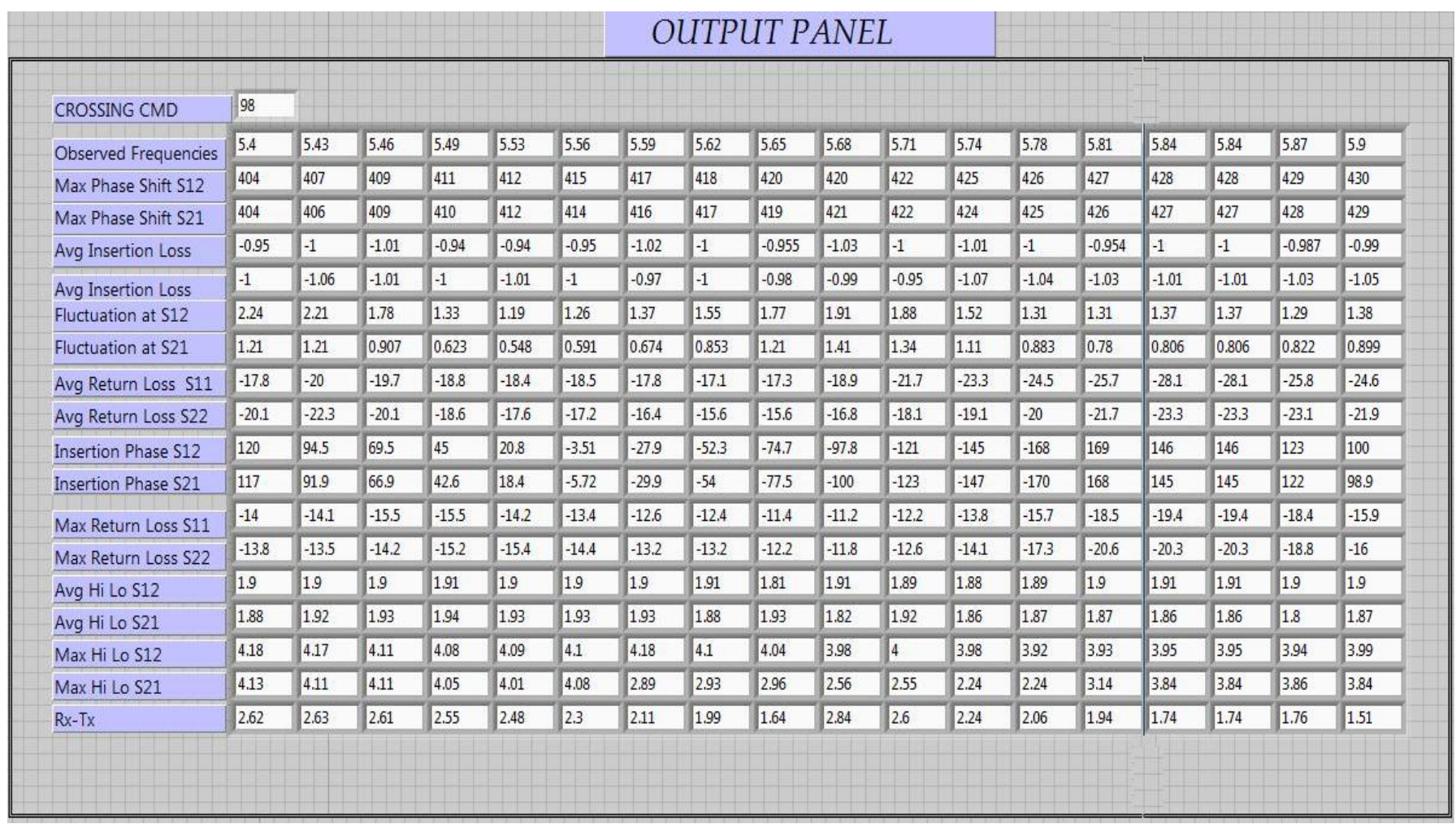

Fig 6b: Output conclusive summary of measured R.F. parameter

For the industrial purpose the result can be displayed also

in graphical form as shown in fig 7 given below: 

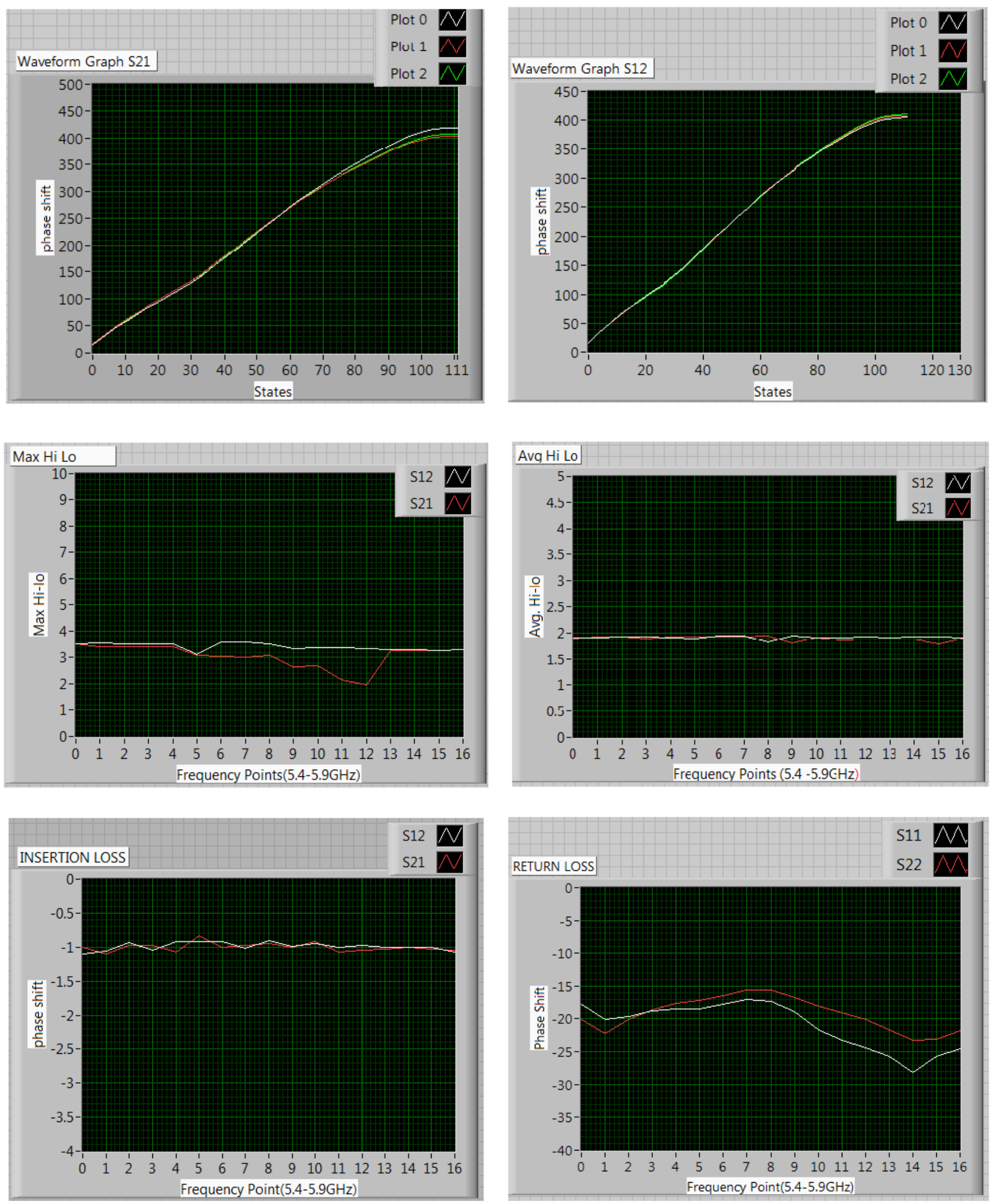

Fig 7: Graphical representation of Measured RF parameters of the Dual mode phase shifter 


\section{CONCLUSIONS}

This paper present the design and development of ATP program for automated characterization and testing of Dual mode phase shifter. The results has been verified and tested. Using this software the bulk production of DMPS will become more feasible.

The LabVIEW as a graphical programming language makes future up gradation less time consuming due to flexibility in design as compare to complex text based code language.

This automatic test procedure software enables fast,efficient measurements, appropriate data processing and orderly storage of huge measured data in the evaluation of C-band Dual Mode Phase Shifters.

\section{ACKNOWLEDGEMENT}

The authors would like to acknowledge the Director, SSPL for his constant interest and encouragement during the development process.

\section{REFERENCES}

[1] Charles R. Boyd, "A Dual-Mode Latching, Reciprocal Ferrite Phase Shifter", IEEE Transactions on Microwave Theory and Techniques (Volume:18, Issue: 12 ), Dec 1970. [2] K K Jain, Kirti Bansal, Meenakshi Aggarwal, B S Matheru," Automatic Test Procedure for RF Characterization of C-band Rotary Field Phase Shifter(Volume ) July 15, 2010.

[3] C. R. Boyd,"Accuracy Study for A Moderate Production Quantity Of Reciprocal Ferrite Phase Shifters" IEEE Transactions on Microwave Theory and Techniques ,1979

[4] Basil Hamed," Design \& Implementation of Smart House Control Using LabVIEW ", International Journal of Soft Computing and Engineering (IJSCE) ISSN: 2231-2307 Volume-1, Issue-6, January 2012

[5] Pavel V. Nikitin," LabVIEW-Based UHF RFID Tag Test and Measurement System", IEEE transactionon industrial electronics, VOL. 56, no. 7, july 2009

[6] Alan J. Fenn, Donald H. Temme, William P. Delaney, and William E. Courtney," The Development of Phased-Array Radar Technology"Volume 12, Number 2, 2000 Linckon Laboratory Journal

[7] LabVIEW User Manual, April 2003 Edition, National Instruments.

\section{BIOGRAPHIES}

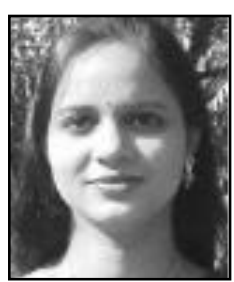

Ms. Kirti Bansal did her B.tech. in electronics and communication engineering from UP Tech University in 2007 batch. Now she is in microwave group in SSPL Lab, DRDO, Delhi. She has made invaluable contribution in the successful recent development of C- band Rotary Field Phase Shifter. And currently, she has been working on the development microcontroller based digital driver of dual mode phase shifter.

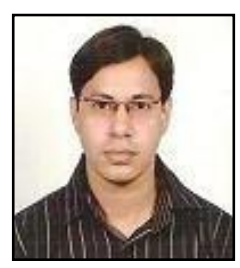

Raj Kr Gautam completed his B.Tech. in electronics and communication engineering from Indian Institute of Technology, Roorkee in 2003 batch. During his 12 year experience he worked in MMIC, Microwave field and also guided various undergraduate trainee students. Presented work is carried out in Microwave Group at SSPL. His involvement is in development of C- band RFPS and microcontroller based driver design of C-band DMPS.

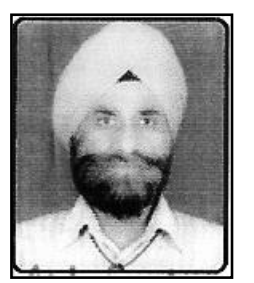

BS Matheru did M.Sc.(Physics) with Electronics followed by M.Tech. in Microwave Electronics in 1980. Thereafter he served in OCS \& WPC/WMO, Ministry of Communications for two and one year respectively. Since 1984, he has been working in Solid State Physics Laboratory, DRDO, in Microwave ferrite based device areas e.g. C- band Dual Mode Phase Shifters, S- band high power Rotary Field Phase Shifter, C- band Rotary Field Phase Shifter, microcontroller based new driver design for C-band DMPS, characterisation of ferrite/dielectric materials, digital drivers and control devices. 